\title{
Evaluation of scaling-up of HPV self-collection offered by community health workers at home visits to increase screening among socially vulnerable under-screened women in Jujuy Province, Argentina
}

\author{
Silvina Arrossi ${ }^{* *}$, Melisa Paolino², Laura Thouyaret ${ }^{2}$, Rosa Laudi $^{2}$ and Alicia Campanera ${ }^{3}$
}

\begin{abstract}
Background: Self-collection has been proposed as a strategy to increase cervical screening coverage among hard-to-reach women. However, evaluations of the implementation of this strategy on a large scale are scarce. This paper describes the process and measurement of the scaling-up of self-collection offered by community health workers during home visits as a strategy to reach under-screened women aged 30+ with public health coverage, defined as the target women.

Methods: We used an adaptation of the Health System Framework to analyze key drivers of scaling-up. A content analysis approach was used to collect and analyze information from different sources. The RE-AIM (Reach, Effectiveness, Adoption, Implementation, and Maintenance) model was used to evaluate the impact of the strategy.

Results: HPV self-collection was scaled-up in the province of Jujuy in 2014 after a RCT (Self-collection Modality Trial, initials EMA in Spanish) was carried out locally in 2012 and demonstrated effectiveness of the strategy to increase screening uptake. Facilitators of scaling-up were the organizational capacity of the provincial health system, sustainable funding for HPV testing, and local consensus about the value of the technology. Reach: In 2014, 9\% $(2983 / 33,245)$ of target women were screened through self-collection in the Jujuy public health sector. Effectiveness: In 2014, 17\% ( $n=5657 / 33,245)$ of target women were screened with any HPV test (self-collected and clinician-collected tests) vs. $11.7 \%(4579 / 38,981)$ in 2013 , the pre-scaling-up period $(p<0.0001)$. Implementation: Training about the strategy was provided to $84.2 \%(n=609 / 723)$ of total community health workers (CHWs). Of 414 HPV+ women, $77.5 \%(n=320)$ had follow-up procedures. Of 113 women with positive triage, $66.4 \%(n=75)$ had colposcopic diagnosis. Treatment was provided to $80.7 \%$ of CIN2+ women $(n=21 / 26)$. Adoption: Of trained CHWs, 69.3\% ( $n=422 / 609)$ had at least one woman with self-collection; $85.2 \%(n=315 / 368)$ of CHWs who responded to an evaluation survey were satisfied with self-collection strategy. Maintenance: During 2015, 100.0\% (723/723) CHWs were operational and $63.8 \%(461 / 723)$ had at least one woman with self-collection.

(Continued on next page)
\end{abstract}

\footnotetext{
* Correspondence: silviarrossi2020@gmail.com

${ }^{1}$ Centro de Estudios de Estado y Sociedad/Consejo Nacional de Investigaciones Científicas y Técnicas, Sánchez de Bustamante 27, Buenos Aires 1193, Argentina Full list of author information is available at the end of the article
} 
(Continued from previous page)

Conclusions: The strategy was successfully scaled-up, with a high level of adoption among CHWs, which resulted in increased screening among socially vulnerable under-screened women.

Keywords: Cervical cancer prevention, Self-collection HPV test, Argentina, Health System Framework, Implementation research, RE-AIM Framework, Scaling-up

\section{Background}

A key component of cervical cancer prevention programs is the achievement of high screening levels, particularly among poor women with low access to the health system. It is well known that human papillomavirus (HPV) testing has important advantages over the Pap as a screening test: high sensitivity (over 90\%) [1] and high negative predictive value [2]. Very importantly, through self-collection, HPV testing can reduce barriers to screening and increase coverage [3], especially among hard-to-reach women who are at higher risk of cervical cancer [4]. The method is highly accurate [5], acceptable to women, and effective to increase screening uptake [6-10]. However, this evidence comes mainly from controlled research studies. Although in the last years there has been an increasing recognition of the importance of documentation and analysis of how scaling-up experiences are initiated, led, and monitored [11], very little evidence exists about how to scale-up HPV self-collection, potentially jeopardizing its successful integration into cervical cancer prevention programs.

In 2012, self-collection was implemented in the province of Jujuy as part of theEMA study (Self-collection Modality Trial, initials EMA in Spanish), a mix-method research study $[9,12,13]$ that included a randomized controlled trial (RCT) to evaluate effectiveness of HPV self-collection offered by community health workers (CHWs) at home visits to increase screening uptake [9]. The intervention resulted in a fourfold increase in screening uptake (from $20.2 \%$ to $85.9 \%$ ), demonstrating that offering the strategy was effective to improve cervical screening uptake [9]. Based on these findings, in 2014, selfcollection of HPV testing was scaled-up to the whole province. The project was led by the National Cancer Institute, Argentina, in collaboration with the Jujuy Ministry of Health.

In this article, we report results of a study carried out to evaluate the scaling-up of HPV self-collection using implementation research methods. For this, we used an adaptation of the Health System Framework $[14,15]$ (HSF) and the Reach, Effectiveness, Adoption, Implementation, and Maintenance (RE-AIM) model for evaluation [16]. The specific aims of the study were to (1) identify key drivers of the scaling-up of HPV self-collection; (2) evaluate if selfcollection was effective to increase screening uptake among under-screened women when implemented on a larger scale; (3) evaluate if the self-collection strategy was accepted and adopted by CHWs; and (4) identify main implementation barriers. Thus, the study provides evidence about the scaling-up process that is key for countries considering incorporation of HPV testing into their cervical cancer control programs.

\section{Methods}

\section{Intervention context}

A detailed description on the context can be found elsewhere [9, 17], but briefly in Argentina, the population not covered by the social security sector (workers of the informal economy and their families) has public health coverage; health care is provided free of cost. Jujuy is located in Northwest Argentina; 45.2\% of population has public health coverage [18]. Its public health system includes a main hospital and 270 primary health care (PHC) centers.

The PHC system employs more than 700 full-time CHWs who visit around 110,000 households (70\% of total provincial households) [18] twice yearly for healthrelated tasks including promotion of HPV testing at health centers. Non-visited households are mainly those located in middle-high and high income urban areas. During the visits, CHWs collect socio-demographic and health data of the household and its members, which is used to annually update the PHC census.

A national screening information system (SITAM) [19] registers data on screening tests and diagnostic procedures from women attending opportunistically public health centers, as there is no call and re-call system.

Scaling-up of self-collection was nested into the Jujuy Demonstration Project (JDP) carried out between 2011 and 2014 (see Fig. 1) to evaluate the introduction of the HPV test as the primary screening method for women

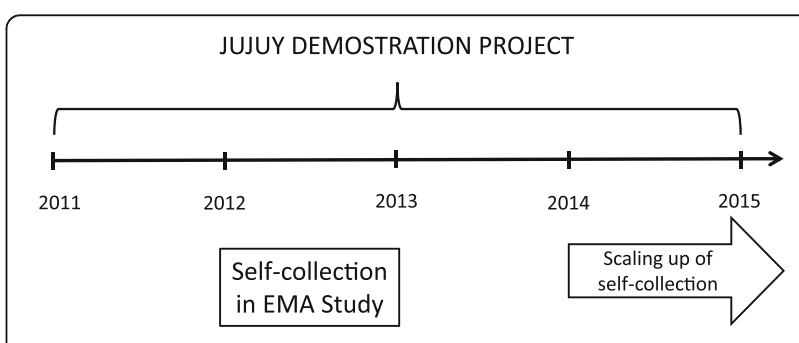

Fig. 1 Timeline of the Jujuy Demonstration Project 
aged 30+ attending public health services [17]. Cytology is used as the triage test of HPV+ women.

\section{The intervention}

In Jujuy, in 2014, the strategy of self-collection offered by CHWs during home visits was scaled-up for all women with public health coverage and without a screening test in the last 5 years $(n=33,245)$. To implement the strategy, health authorities decided to involve all 723 CHWs belonging to $\mathrm{PHC}$ system. The core components of the intervention scaled-up included the offer of self-collection during home visits by CHWs, sample handling and transportation, follow-up of HPV+ women and treatment if needed, and training of CHWs, as described below.

\section{Target population}

Target population were all women aged $30+$ included in the PHC census, not screened in the previous 5 years (under-screened women) with public health coverage.

\section{Offer of self-collection}

Self-collection was offered by CHWs during home visits throughout the second half of 2014. During the visit, CHWs provided women with information about cervical cancer prevention and HPV testing and then offered them HPV self-collection, followed by a 10-min step-by-step explanation on how to perform it using communication support material (Additional file 1). Self-samples were collected using the Qiagen cervical sampler during the $\mathrm{CHW}$ visit. Pregnant women were not offered self-collection.

\section{Sample handling and transportation to PHC centers}

CHWs labeled collectors with the woman name and the national unique identifier number and transported specimens at room temperature to PHC centers: from here they were sent to the provincial HPV laboratory by the health center. Samples were tested for 13 high-risk HPV types using hybrid-capture 2, following manufacturer's instructions. Specimens were not processed if received
$>14$ days after collection, without liquid, brush, or identification data (discarded samples).

\section{Follow-up and treatment}

According to guidelines [20], self-collected HPV+ women were referred to cytology triage. HPV+ women with normal cytology were advised to repeat test at 18 months. Women with abnormal cytology (atypical squamous cells of undetermined significance; atypical cells cannot rule out high-grade squamous intraepithelial lesion; high-grade squamous intraepithelial lesions; and cancer) were referred to colposcopy, and biopsy if needed. Biopsies were reported according to cervical intraepithelial neoplasia (CIN) terminology. Identified cases of CIN2+ were treated according to standard protocols (loop electrosurgical excision procedure (LEEP) for CIN). HPV-negative women were advised to repeat screening within 5 years following national recommendations.

\section{Training of CHWs}

From April to June 2014, national and provincial team members led 18 workshops aimed at training of all 723 CHWs. Description of structure and content of workshops are shown in Table 1. They included expert presentations, discussions in small groups, and role playing to recreate different scenarios during the offer of self-collection. At the end of each training workshop, CHWs were provided with the list of women to be offered the test, self-collection kits, and other materials needed for the offer (i.e., educational materials). If needed, additional collectors/materials could be requested to health centers or the provincial program headquarters.

By the end of 2014, once the round of home visits was finished, an evaluation workshop was carried out with all CHWs. During this activity, CHWs were invited to complete a self-administered questionnaire (SEAQ) to elicit their views and opinions about the incorporation of HPV self-collection as a programmatic activity. At the beginning of 2015, a refresher training was provided to all CHWs.

Table 1 Description of training workshops

\begin{tabular}{|c|c|}
\hline Sections & Contents \\
\hline $\begin{array}{l}\text { Project } \\
\text { background }\end{array}$ & Information on the EMA study and scaling-up in a programmatic context \\
\hline Cervical cancer & Scientific data on cervical cancer and its relation with HPV \\
\hline HPV testing & Basic information on HPV testing as primary screening strategy for cervical cancer prevention \\
\hline HPV self-collection & $\begin{array}{l}\text { HPV self-collection: clinician vs. self-collected tests, step-by-step self-collection take-up, self-collection results understanding, and } \\
\text { follow-up of HPV+ women }\end{array}$ \\
\hline $\begin{array}{l}\text { Communication } \\
\text { skills }\end{array}$ & Communication skills to conduct the educational talk (instruct women on how to perform self-collection) \\
\hline $\begin{array}{l}\text { Logistical } \\
\text { procedures }\end{array}$ & List of women to be offered self-collection, collectors, and training on sample labeling and transportation \\
\hline
\end{tabular}




\section{Evaluation of scaling-up}

To answer the different questions addressed by the study, we used a combination of methods:

1. Analysis of the scaling-up process. For this analysis, we used an adaptation of the HSF $[14,15]$, which includes contextual interconnected factors that are considered key drivers for successful scaling-up [21]. The HSF integrates six functions, or building blocks (service delivery, health workforce, information, technology, funding, stewardship) that influence system performance. We have re-configured and simplified these functions to incorporate an organizational dimension, which is a key factor for cervical cancer prevention program effectiveness [22]. Thus, this adaptation presents four dimensions for analysis, instead of the original six, as service delivery, health care workforce, and information have been integrated into the dimension organizational capacity, as follows (Fig. 2).

(a) Stewardship: Policy support; existence of policy guidelines/norms that promote alignment with strategic health goals; partnerships for collaboration

(b) Organizational capacity: Capacity of the health system to implement the intervention in all stages of the screening/diagnoses/treatment continuum, including facilities and health workforce; and availability of health information systems

(c) Technology/strategy: Acceptability, effectiveness, and consensus about the value of the technology/ strategy

(d)Funding: availability of sustainable funding
The analysis was based on content analysis of program reports (information sheets, power point presentations, etc.) and observation derived from our participation in the policy definition. The analysis was verified by triangulation across the different sources. Also, preliminary results were presented and discussed in a meeting of provincial policy makers and health providers, as well as in the final evaluation workshop, to discuss and verify research findings.

2. Analyses of dimensions proposed by the RE-AIM model $[16,23]$.

\section{Reach}

We measured the percentage of under-screened women with public health coverage with self-collected tests/total target population. Additionally, we compared target women with HPV self-collection and the total target population according to their age distribution using simple frequencies and percentages.

\section{Effectiveness}

We evaluated two outcomes: (1) The increase in screening uptake among the target population. For this, we compared the difference between the percentage of the target population who was HPV-tested (both with self-collection and clinician-collected tests at health centers)/total target women in 2014 (post scale-up) and the percentage of the target population screened at health centers/total target women in 2013 (the year previous to self-collection introduction). (2) The increase in the proportion of total screened women who were from the target population. For this, we compared the pre and post scaling-up percentage of screened women who were from the target

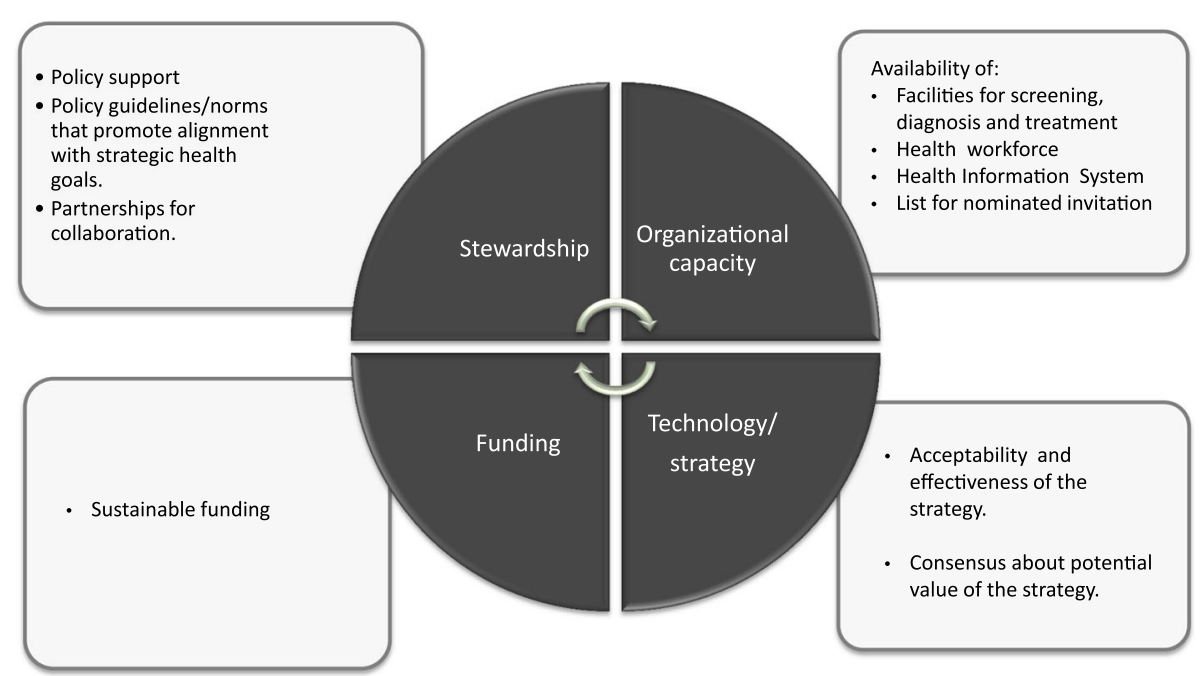

Fig. 2 Health System Framework for analysis of cervical cancer prevention 
population/total screened women. Pre and post scalingup percentages were compared using chi-square tests of association, and statistical significance was established at $p<0.05$.

\section{Adoption}

Three outcomes were measured: (1) percentage of adopters: number of CHWs with at least one self-collected sample registered in SITAM/total trained CHWs; (2) average number of women with self-collected tests per CHW; and (3) percentage of CHWs satisfied with the self-collection strategy/total CHWs who answered the SEAQ.

\section{Implementation}

Three outcomes were measured: (1) percentage of trained CHWs/total CHWs; (2) percentage of CHWs who reported at least one problem to obtain materials (kits, leaflets)/total CHWs who answered the SEAQ; and (3) percentage of discarded samples registered in lab records/total number of self-collected tests.

Additionally, for this dimension, we analyzed follow-up in the target population: (1) percentage of HPV+ women with follow-up procedures, HPV+ women were considered to have received follow-up if they had at least one of the following procedures, independently of the protocol recommendation: cytology triage, colposcopy, or second HPV negative test; (2) percentage of HPV+ women with cytology triage (as per protocol); (3) percentage of women with positive triage who had colposcopic diagnosis/total women with positive triage; and (4) percentage of treated women/women with CIN2+ lesions.

\section{Maintenance}

One outcome was measured: percentage of trained CHWs with at least one self-collected sample registered in SITAM in 2015/total trained CHWs.

\section{Databases}

Three databases were built specifically for the study. First, we linked the 2013 PHC census to the SITAM database to draw up the list of target women to be offered selfcollection. After field work, we linked the list of target women to SITAM to extract data on HPV testing, triage, diagnosis, and treatment, using each woman's national identity document number. To calculate the percentage of screening uptake in 2013, we linked the 2012 list of target population to the SITAM database. We also built a specific database with CHWs information, including sex, type of setting, and participation in training workshops.

Finally, we used the SEAQ to elicit CHWs views about the offer of self-collection, specifically on their satisfaction with the strategy, and problems during the implementation. CHWs were asked to fill the SEAQ during the final evaluation workshop carried out at the end of the health round. It was anonymous, and participation was voluntary; a member of the research team explained its objectives and how to complete it.

\section{Results}

Results of the analysis based on the Health System Framework are presented below.

\section{Stewardship}

The EMA study was a research project carried out collaboratively by national and provincial Ministries of Health (MoHs). Expansion of the strategy was discussed by the National Program of Cervical Cancer Prevention (NPCC) with provincial authorities by the end of 2013 after results from the EMA study were available. Scaling-up was considered key to reach provincial screening coverage objectives [17]. A proposal was presented to and approved by the Jujuy Demonstration Project Scientific Advisory Committee (Advisory Committee), which included members from main medical scientific societies, international health organizations (Pan American Health Organization, International Agency for Research on Cancer, and United Nations Population Fund), and women organizations such as the Women National Council. Thus, the decision to scale-up was agreed in a context of high support by national and provincial health authorities, and international health organizations.

Next, core components and algorithm of the scaling-up (described above) were defined on a national/provincial management round-table. Their members met at least twice during the implementation phase to monitor and evaluate process results, as well as to identify emerging problems. Introduction of HPV self-collection was incorporated into provincial norms and regulations, and national protocols [20].

\section{Organizational capacity}

The organizational capacity of the province was considered adequate. Jujuy has a wide network of PHCs, and $\mathrm{CHWs}$ visit houses twice a year as a regular, programmatic activity. A referral network for triage, diagnosis, and treatment was already in place, and health providers had received training on HPV testing in the context of the JDP initiated in 2011 [17]. The province runs the first country public HPV laboratory, with more than 50,000 samples processed between 2012 and 2014. A team of two navigators identifies women with abnormal Pap smears to provide support in cases where women have difficulties to complete diagnosis/treatment. However, no specific support is provided to $\mathrm{HPV}+$ women who need to be triaged. SITAM, the information system for monitoring and evaluating screening activities [19], was already in place, with registry of all women being screened at public health institutions. SITAM was used 
to build the nominated list of target women to be offered self-collection by CHWs.

\section{Technology/strategy}

Local evidence indicated that the strategy was both acceptable to women $[9,12]$ and CHWs [13], and effective at increasing screening uptake [9], in agreement with studies carried out in other countries and settings. Local qualitative research carried out by our team had also shown its high acceptability among provincial health professionals, especially as a strategy to reach under-screened women (Arrossi, unpublished observations). The Advisory Committee also acknowledged the strong potential of HPV self-collection to increase uptake although their members expressed concern about its relatively lower detection rate when compared to clinician-collected tests [5]. As a result, the Advisory Committee strongly recommended HPV self-collection to screen hard-to-reach women. The Advisory Committee also discussed and agreed on the proposed algorithm for HPV self-collection, which implied a modification of the algorithm used in the EMA RCT [9]. In effect, due to the relatively low performance of colposcopic diagnoses in the absence of a Pap smear result found in that project (Arrossi, unpublished observations), it was decided that HPV+ women would be referred to cytology triage.

\section{Funding of scaling-up}

Funding of the scaling-up was evaluated as sustainable, as it was implemented in the context of the JDP, with funding by the national and provincial MoHs. The HPV test was provided by the national $\mathrm{MoH}$ as part of the JDP. In addition, the EMA study was carried out in a programmatic context, with provincial health providers and infrastructure funded through the regular budget. Therefore, a decision was made by health authorities to guarantee the funding needed to scale-up the strategy. Triage, diagnosis, and treatment of HPV+ women were provided by public health institutions, free of cost for women, using the provincial referral network, which has been described elsewhere [17]. Communication materials used for the offer of self-collection were those used in the EMA RCT and provided to the province by the NPCC, as part of the regular provision of communication materials by the national $\mathrm{MoH}$ to provinces. SITAM is run and maintained by the national $\mathrm{MoH}$.

\section{RE-AIM measurement Reach}

In 2014, 2983 women from the target population (9\%) were screened with self-collected tests. Compared to the target population (Table 2), more women with selfcollected tests were aged $35-44$ (37.0 vs. $32.8 \%)$ and less women were aged $65+$ ( 4.6 vs. $8.7 \%)$.
Table 2 REACH: Comparison of women with self-collected tests with total target population, 2014

\begin{tabular}{|c|c|c|c|c|c|}
\hline & \multicolumn{2}{|c|}{$\begin{array}{l}\text { Target population with } \\
\text { self-collected test }\end{array}$} & \multicolumn{2}{|c|}{ Total target population } & \multirow[t]{2}{*}{$p$ value } \\
\hline & $n$ & $\%$ & $n$ & $\%$ & \\
\hline \multicolumn{6}{|l|}{$\overline{\text { Age }}$} \\
\hline $30-34$ & 677 & 22.8 & 7827 & 23.5 & \multirow[t]{6}{*}{$p<0.001$} \\
\hline $35-44$ & 1105 & 37.0 & 10,908 & 32.8 & \\
\hline $45-54$ & 615 & 20.6 & 6580 & 19.8 & \\
\hline $55-64$ & 448 & 15.0 & 5033 & 15.2 & \\
\hline $65+$ & 138 & 4.6 & 2897 & 8.7 & \\
\hline Total & 2983 & 100.0 & 33,245 & 100.0 & \\
\hline
\end{tabular}

${ }^{*}$ Chi-square test, statistical significance was established at $p<0.05$

\section{Effectiveness}

In 2014, 12,778 women aged 30+ were HPV-tested in the Jujuy public health sector, $44.3 \%(n=5657 / 12,778)$ were target population, a 38\% increase from 2013 (32.1\%; $n=4579 / 14,272 ; p<0.0001)$ (Table 3). Of the target population, $17.0 \% \quad(n=5657 / 33,245)$ were HPVtested with any HPV test (2983 with self-collected tests and 2674 with clinician-collected tests at health centers). This is a $45 \%$ increase in screening uptake of the target population relative to pre scale-up level in 2013 with clinician-collected tests at health centers $(11.7 \%$; $n=4579 / 38,981 ; p<0.0001)$.

\section{Adoption}

In total, 609/723 (84.2\%) CHWs were trained; $69.3 \%$ $(n=422 / 609)$ of them had at least one woman with a self-collected sample registered in SITAM; 62.6\% ( $n=$ $381 / 609)$ if only women from the target population are considered (Table 3). The average number of women with self-collected samples per $\mathrm{CHW}$ was 11 (range 1-74), and eight if only screening in the target population is considered (range 1-31).

Of the $368 \mathrm{CHWs}$ who completed the SEAQ, 85.6\% of $(n=315)$ mentioned that they were satisfied with the self-collection strategy (Table 3 ).

\section{Implementation}

Training about the strategy was provided to $84.2 \%(n=609)$ of total CHWs (Table 3). When asked about problems during implementation, $20.7 \%$ reported having at least one problem to obtain collectors, leaflets, or materials needed for the offer. Percentage of discarded samples at the laboratory was $0.9 \%(29 / 2983)$.

\section{Adherence to follow-up, diagnosis, and treatment}

Of the 2983 target women with self-collected tests, 414 (13.9\%) were HPV+ (Fig. 3). Of these, 77.5\% ( $n=321)$ had follow-up procedures: 282 (68.1\%) had cytology triage, as per protocol: 158 were normal, 99 abnormal and there 
Table 3 RE-AIM measurement

\begin{tabular}{|c|c|c|c|}
\hline RE-AIM dimension & Question & Outcome & Value \\
\hline Reach & $\begin{array}{l}\text { What are the characteristics of women } \\
\text { reached by the strategy? }\end{array}$ & $\begin{array}{l}\text { Under-screened women with public health coverage } \\
\text { with self-collected test (2014) }\end{array}$ & $9.0 \%$ \\
\hline \multirow[t]{4}{*}{ Effectiveness } & \multirow[t]{4}{*}{$\begin{array}{l}\text { Is the strategy effective to increase } \\
\text { screening uptake in target population? }\end{array}$} & $\begin{array}{l}\text { Screened women from target population, } \\
\text { post-intervention (2014) }\end{array}$ & $44.3 \%$ \\
\hline & & $\begin{array}{l}\text { Screened women from target population, } \\
\text { pre-intervention (2013) }\end{array}$ & $32.1 \%$ \\
\hline & & $\begin{array}{l}\text { Target population with screening uptake, } \\
\text { post-intervention (2014) }\end{array}$ & $17.0 \%$ \\
\hline & & $\begin{array}{l}\text { Target population with screening uptake, } \\
\text { pre-intervention (2013) }\end{array}$ & $11.7 \%$ \\
\hline \multirow[t]{3}{*}{ Adoption } & \multirow[t]{3}{*}{$\begin{array}{l}\text { Is the self-collection strategy accepted } \\
\text { and adopted by CHWs? }\end{array}$} & $\begin{array}{l}\text { CHWs with at least one self-collected test registered } \\
\text { in SITAM }\end{array}$ & $69.3 \%$ \\
\hline & & $\begin{array}{l}\text { CHWs with at least one self-collected test in target } \\
\text { population registered in SITAM }\end{array}$ & $62.6 \%$ \\
\hline & & $\begin{array}{l}\text { CHWs that mentioned that they were satisfied with } \\
\text { self-collection strategy }\end{array}$ & $85.6 \%$ \\
\hline \multirow[t]{7}{*}{ Implementation } & \multirow{7}{*}{$\begin{array}{l}\text { To what extent the intervention was } \\
\text { implemented as intended? }\end{array}$} & Trained CHWs/total CHWs & $84.2 \%$ \\
\hline & & $\begin{array}{l}\text { CHWs who reported at least one problem to obtain } \\
\text { materials }\end{array}$ & $20.7 \%$ \\
\hline & & $\begin{array}{l}\text { Discarded samples registered in lab records/total number } \\
\text { of self-collected tests }\end{array}$ & $0.9 \%$ \\
\hline & & Follow-up in TP & \\
\hline & & HPV+ with follow-up procedures & $77.5 \%$ \\
\hline & & Triage + women with colposcopy & $66.4 \%$ \\
\hline & & Women with $\mathrm{CIN} 2+$ with registered treatment & $80.7 \%$ \\
\hline Maintenance (1 year) & $\begin{array}{l}\text { Is it possible to sustain the intervention } \\
\text { over time? }\end{array}$ & $\begin{array}{l}\text { Trained CHWs with at least one self-collected test registered } \\
\text { in SITAM in } 2015\end{array}$ & $63.8 \%$ \\
\hline
\end{tabular}

TP target population, CHWs community health workers

were no available results for $25 ; 14$ had only colposcopy, and 25 had a second negative HPV test. Total women with colposcopic diagnosis were 75 (66.4\% of women with positive triage), including 14 colposcopies without cytology result (34 normal, 35 abnormal; 6 women had colposcopies at private services without available results).

Among women with abnormal colposcopy, 34 had biopsies performed at public health services and had results in SITAM. Among them, 26 had CIN2+ (detection rate $0.9 \%, n=26 / 2983) ; 21$ of these $(80.7 \%)$ received treatment (follow-up until June 30, 2016).

\section{Maintenance}

In the second year of the scaling-up process (2015), 723 (100.0\%) CHWs were operational: all 609 CHWs trained in 2014 and 114 incorporated and trained in 2015. In that year, $63.8 \%(461 / 723)$ had at least one woman with a self-collected test registered in SITAM (Table 3).

\section{Discussion}

Increasing screening uptake among hard-to-reach women is a major critical factor for cervical cancer control. Using the RE-AIM model and the HSF, we analyzed the scaling- up of HPV self-collection offered by CHWs at home visits, in the province of Jujuy, Argentina. The strategy resulted in a $45 \%$ increase in screening of under-screened, socially vulnerable population. Results also showed that most CHWs adopted the strategy, with few implementation problems. Leadership and partnership of national and local health decision makers, governmental funding, an adequate organizational capacity of the health system, and consensus about the potential value of the strategy were identified as key drivers of the scaling-up.

This is, to our knowledge, the first study analyzing scaling-up of a HPV self-collection strategy using implementation science methods. Utilization of the RE-AIM framework allowed us to include in the analysis the five factors that more completely characterize the public health impact of an intervention carried out in real-world environments [16]. In addition, the HSR framework guided the evaluation to identify key drivers of the scaling-up process. Thus, this study can serve as a model of how implementation science can be used to plan, conduct, and evaluate interventions that are implemented on a large scale in programmatic, real-world contexts. Stewardship refers to the policy environment in which the implementation 


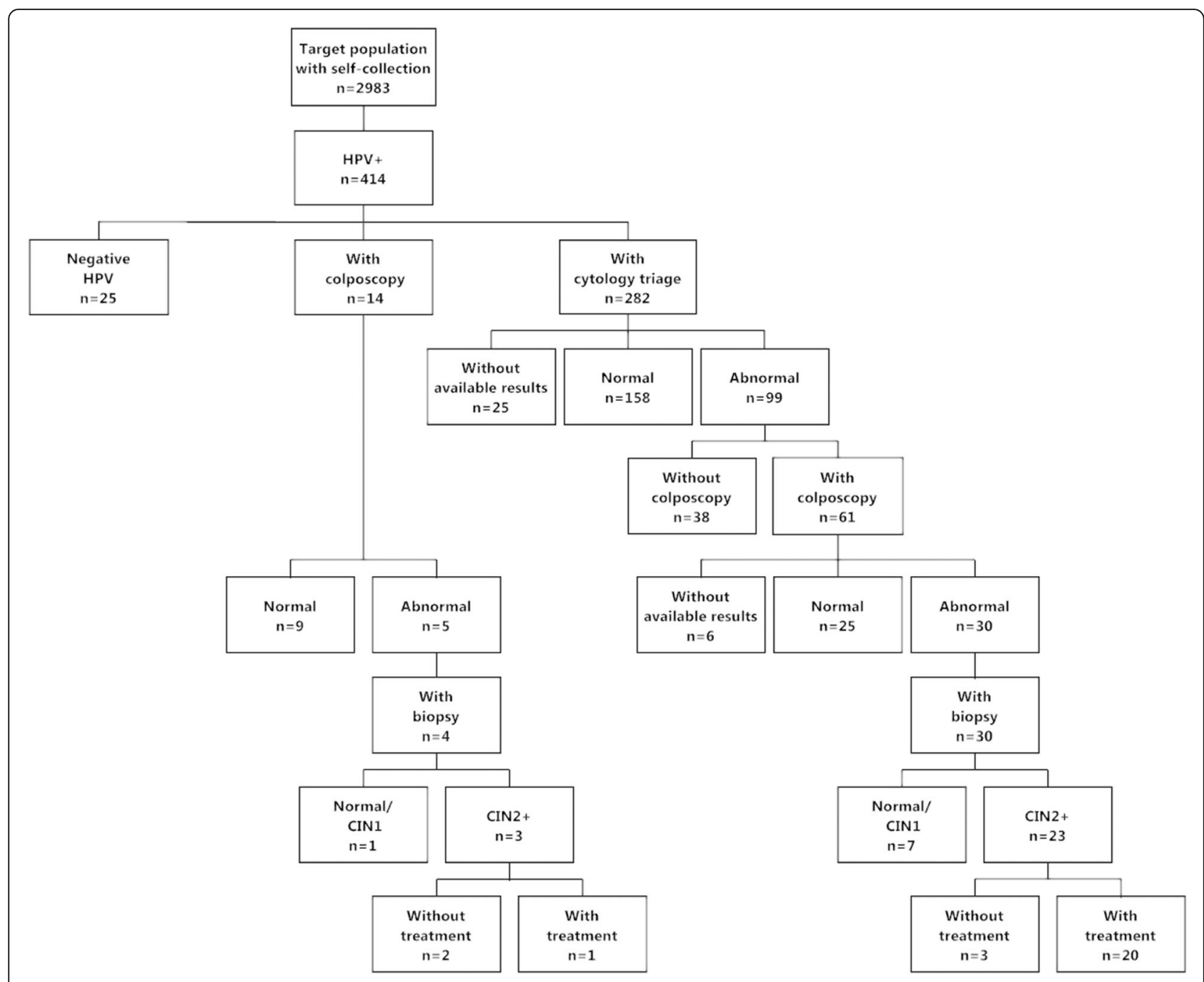

Fig. 3 Follow-up of HPV+ women among target population. Jujuy 2014

of a health strategy is made possible, through three main dimensions: formulating health policy, exerting influence, and collecting and using intelligence [24]. In our study, scaling-up of HPV self-collection as part of a national demonstration project to introduce HPV testing as primary screening [17] provided governmental stewardship in a context of high policy support. This was reinforced by involvement of the scientific community and other main health and women organizations, which ensured alignment of objectives and goals. Another key factor was that scaling-up was based on the EMA study, a research project carried out in a programmatic context and run collaboratively between the national and provincial health authorities. Incorporating research into implementation, country ownership, and political will have been identified as factors associated with faster diffusion of an innovation $[25,26]$. By making use of legal, regulatory, and policy instruments to steer health system performance [24], stewardship bears a strong relationship to the concept of regulation [27]. In our study, inclusion of the strategy into national and provincial norms and protocols was also identified as a key enhancer of the scaling-up.

Integrating activities into existing health systems has been identified in the literature as an important factor for successful scaling-up [28]. In this case, expansion followed the EMA study, using the provincial organizational capacity. Health system weaknesses contributing to poor health outcomes include poor management and a weak PHC system [29]. In Jujuy, the PHC system is well developed, with almost $700 \mathrm{CHWs}$ visiting houses twice a year. The health system included the network of diagnosis and treatment units and the first public HPV laboratory of the country. Lack of information systems is also a key problem affecting cancer control programs [30]. The use of SITAM allowed not only to closely monitor and evaluate the scaling-up but also to build the target population list, facilitating identification and contact with under-screened women. 
Securing sustainable funding for large-scale implementation can be a major challenge. Many pilot or research studies are funded by non-governmental national or international organizations, which results in funding very often ending with the pilot study [31]. Also, interventions proved efficacious in research studies carried out using industry-donated technology might be difficult to scale-up once the research is finished [32]. All funding for this project was provided by the regular mechanisms of public health system financing. Thus, scaling-up was guaranteed and stewardship strengthened, sending also a strong message to the community about the government commitment with its implementation in the context of the national policy for cervical cancer prevention.

The study showed increased screening among hard-toreach women compared to what was attained the year previous to the scaling-up. It is possible that part of the population tested with clinician-collected tests were women offered self-collection by CHWs who preferred to get screened at health centers. This was observed in the RCT of the EMA study, where 15\% of women contacted by $\mathrm{CHWs}$ preferred clinician-collected tests [9]. However, our study design did not allow measuring the impact of offering HPV self-collection on screening performed at health centers. It is neither possible to know if women reached by HPV self-collection would have eventually been screened at health centers, but in any case, the strategy is at least advancing the date of screening of these women. It is possible as well that the women reached by self-collection would have never been screened at all. Evidence shows that under-screened women have a higher risk of developing cervical cancer [33]. In addition, women with public health coverage are a socially vulnerable population, with over-representation among women with cervical cancer in Argentina [34]. If the strategy is maintained, and its pace, $80 \%$ of these hard-to-reach, high-risk women, could be screened in 5 years with high potential impact on cervical cancer control among this population.

Adoption has been described as the intention to employ an innovation or evidence-based practice [35]. Our study showed an adequate level of adoption of the strategy by CHWs, with $69 \%$ of trained $\mathrm{CHWs}$ with a self-collected test registered in SITAM. This is probably an under-estimation of the level of adoption as some CHWs might have adopted the strategy without being successful in the offer of self-collection, due to women characteristics or circumstances beyond his/her control. That is also a possible explanation to the fact that when we analyze the percentage of CHWs with at least one self-collected sample in the target population, the figure drops to $62 \%$. Most CHWs were satisfied with offering the strategy. A qualitative study on CHWs' experiences about HPV self-collection [13] showed that the possibility of having an active role in cervical cancer prevention activities was a main motivation factor for CHWs.

The implementation dimension in the RE-AIM model refers to the extent to which a program is delivered as intended [16]. In our study, training was delivered to the vast majority of $\mathrm{CHWs}$, and there were almost no discarded samples at the laboratory. Few problems were reported with provision of self-collection materials. Availability of these supplies has a direct impact on the possibility of offering the test, and therefore, an interruption in their provision must be closely monitored. Finding a low frequency of reported issues was considered particularly important, as problems in access to inputs essential to CHWs provision of health services can negatively affect their work [36].

High coverage will not result in a decrease of disease burden if women are not diagnosed/treated. The protocol for scaling-up of self-collection recommended cytology as the triage test. Still there were $14 \mathrm{HPV}+$ women who had colposcopy without a previous cytology, revealing a problem of adherence to the triage protocol which has also been found in another study [3]. In our study, $77.5 \%$ of $\mathrm{HPV}+$ women had follow-up procedures. Compliance with triage is lower than in the EMA RCT [9] and in a Chilean study [7] where $86 \%$ of women had colposcopic referral after an HPV positive test. However, the scalingup protocol included a visit for cytology triage, an additional step not included in the EMA study. In a study carried out in France among non-attenders of low socioeconomic level, $41 \%$ of women had a Pap smear after a positive HPV self-collected test [37]. Adding a visit to under-screened women poses a problem, as very probably the barriers faced by women to get screened at health centers [38, 39] partly account for the loss to cytology triage. This underscores a major limitation of selfcollection followed by cytology. The protocol in place at health centers for clinician-collected samples states that HPV tests and Pap smears must be taken at the same time [17], but this is not possible in a self-collection context. Among women with self-collected tests, CIN2+ detection rate was $0.9 \%$. This rate is lower than the one reported in the literature [5], and specifically in the EMA RCT [9], most probably due to the loss to follow-up, both at triage and colposcopic diagnosis.

Our study has a main limitation. We were not able to measure how many women were contacted by $\mathrm{CHWs}$; therefore, we cannot evaluate how successful they were in the offer of self-collection. When we compare the average number of self-collected samples per CWH reported in the EMA RCT with the average obtained during scaling-up of the strategy, we observe that the figure drops (11 vs. 30). This decrease can be explained by several factors. On the one hand, the EMA study included only $\mathrm{CHWs}$ with good evaluation scores, whereas scaling-up was implemented 
with incorporation of all CHWs, independent of their performance scores. Including less-motivated CHWs might have had an impact in the strategy. Also, in the EMA RCT, women at home during the CHW visit were offered selfcollection irrespective of their past Pap screening history, whereas in the scaling-up, CHWs were asked to offer selfcollection to under-screened women with public health coverage. Women who were not at home during the CHW visit could not be offered the strategy, as CHWs visit houses once per round. Besides, the question on satisfaction in the SEAQ was only answered by $61 \%$ of CHWs; therefore, the reported level of satisfaction could be biased.

\section{Conclusions}

We showed that HPV self-collection offered by CHWs at home visits can be adequately scaled-up in programmatic conditions to increase screening of hard-to-reach women. The strategy had a high level of adoption among CHWs, and there were few implementation problems at the screening phase, but obstacles for follow-up and treatment. It is important to devise new strategies and tests to increase triage and diagnosis among HPV+ women. This study provides key evidence for countries and programs planning to incorporate and expand HPV self-collection.

\section{Additional file}

Additional file 1: Communication support material explaining all steps of self-collection take-up. (PDF $1379 \mathrm{~kb}$ )

\section{Abbreviations}

CHWs: Community health workers; CIN: Cervical intraepithelial neoplasia; HPV: Human papillomavirus; JDP: Jujuy demonstration project; LEEP: Loop electrosurgical excision procedure; MoH: Ministry of Health; NPCC: National Program of Cervical Cancer Prevention; PHC: Primary Health Care;

RCT: Randomized control trial; SITAM: National screening information system; TP: Target population

\section{Acknowledgements}

The authors would like to thank the Direction of Primary Health Care, the Jujuy Program on Cervical Cancer Prevention, and CHWs for their support. Also, we would like to thank the team in charge of CHWs training workshops, Maria Antonia Rodriguez for her help with the manuscript and Mariana Curotto for her assistance with the administration and logistics.

\section{Funding}

National Cancer Institute, Argentina, in collaboration with Jujuy Ministry of Health.

\section{Availability of data and materials}

The datasets used in this study are available from the corresponding author on reasonable request.

\section{Author's contributions}

All authors made substantial contributions to the conception and design of the study, acquisition, or analysis of data, and revision of the report. All authors were involved in the interpretation of data and critical revision of the manuscript. SA was the principal investigator and the study coordinator and led the drafting and final editing of the manuscript. MP contributed to the study design, proposed and performed the analysis of the data, and (in consultation with the co-authors) produced the figures and tables. LT contributed to the study design and made substantial contributions to the interpretation of findings and critical revision of the manuscript. AC was a local investigator and made the critical revision of the manuscript. RL contributed to the data analysis and made the critical revision of the manuscript. All authors read and approved the final manuscript.

\section{Competing interests}

The authors declare that they have no competing interests.

Consent for publication

Not applicable.

Ethics approval and consent to participate

Not applicable.

\section{Author details}

${ }^{1}$ Centro de Estudios de Estado y Sociedad/Consejo Nacional de Investigaciones Científicas y Técnicas, Sánchez de Bustamante 27, Buenos Aires 1193, Argentina. ${ }^{2}$ Programa Nacional de Prevención de Cáncer Cervicouterino/Instituto Nacional del Cáncer, Julio A. Roca 781, Buenos Aires 1067, Argentina. ${ }^{3}$ Ministerio de Salud de la Provincia de Jujuy, Av. Italia esq. Independencia, San Salvador de Jujuy 4600, Argentina.

Received: 23 July 2016 Accepted: 3 February 2017

Published online: 13 February 2017

\section{References}

1. Cuzick J, Harbin M, Sankaranarayanan R, Tsu V, Ronco G, Mayrand MH, et al. Overview of human papillomavirus-based and other novel options for cervical cancer screening in developed and developing countries. Vaccine. 2008. doi:10.1016/j.vaccine.2008.06.019.

2. Dillner J, Rebolj M, Birembaut P, Petry KU, Szarewski A, Munk C, et al. Long term predictive values of cytology and human papillomavirus testing in cervical cancer screening: joint European cohort study. BMJ. 2008. doi:10. 1136/bmj.a1754

3. Gök M, Heideman DA, van Kemenade FJ, Berkhof J, Rozendaal L, Spruyt JW, et al. HPV testing on self-collected cervicovaginal lavage specimens as screening method for women who do not attend cervical screening: cohort study. BMJ. 2010. doi:10.1136/bmj.c1040.

4. Lazcano-Ponce E, Lorincz AT, Cruz-Valdez A, Salmerón J, Uribe P,

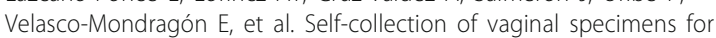
human papillomavirus testing in cervical cancer prevention (MARCH): a community-based randomised controlled trial. Lancet. 2011. doi:10.1016/ S0140-6736(11)61522-5.

5. Arbyn M, Verdoodt F, Snijders PJ, Suonio E, Dillner L, et al. Accuracy of human papillomavirus testing on self-collected versus clinician-collected samples: a meta-analysis. Lancet Oncol. 2014. doi:10.1016/S1470-2045(13)70570-9.

6. Giorgi Rossi P, Marsili LM, Camilloni L, lossa A, Lattanzi A, Sani C, et al. The effect of self-sampled HPV testing on participation to cervical cancer screening in Italy: a randomised controlled trial (ISRCTN96071600). Br J Cancer. 2011. doi:10.1038/sj.bjc.6606040.

7. Léniz J, Barriga MI, Lagos M, Ibáñez C, Puschel K, Ferreccio C. HPV vaginal self-sampling among women non-adherent to Papanicolaou screening in Chile. Salud Publica Mex. 2013:55:162-9.

8. Zehbe I, Moeller H, Severini A, Weaver B, Escott N, Bell C, et al. Feasibility of self-sampling and human papillomavirus testing for cervical cancer screening in First Nation women from Northwest Ontario, Canada: a pilot study. BMJ Open. 2011. doi:10.1136/bmjopen-2010-000030.

9. Arrossi S, Thouyaret L, Herrero R, Campanera A, Magdaleno A, Cuberli M, et al. Effect of self-collection of HPV DNA offered by community health workers at home visits on uptake of screening for cervical cancer (the EMA study): a population-based cluster-randomised trial. Lancet Glob Health. 2015. doi:10.1016/S2214-109X(14)70354-7.

10. Verdoodt F, Jentschke M, Hillemanns P, Racey CS, Snijders PJ, Arbyn M. Reaching women who do not participate in the regular cervical cancer screening programme by offering self-sampling kits: a systematic review and meta-analysis of randomised trials. Eur J Cancer. 2015. doi:10.1016/j. ejca.2015.07.006.

11. Milat AJ, Bauman A, Redman S. Narrative review of models and success factors for scaling up public health interventions. Implement Sci. 2015. doi: 10.1186/s13012-015-0301-6. 
12. Arrossi $S$, Ramos S, Straw C, Thouyaret L, Orellana L. HPV testing: a mixed-method approach to understand why women prefer self-collection in a middle-income country. BMC Public Health. 2016. doi:10.1186/s12889-016-3474-.

13. Curotto M, Barletta P, Paolino M, Arrossi S. La perspectiva de los agentes sanitarios sobre la incorporación programática de la autotoma del test de VPH. Cad Saude Publica. (in press).

14. Leon N, Schneider H, Daviaud E. Applying a framework for assessing the health system challenges to scaling up mHealth in South Africa. BMC Med Inform Decis Mak. 2012. doi:10.1186/1472-6947-12-123.

15. Gilson L, editor. Health policy and systems research: a methodology reader. Geneva: World Health Organization; 2012.

16. Glasgow RE, Vogt TM, Boles SM. Evaluating the public health impact of health promotion interventions: the RE-AIM framework. Am J Public Health. 1999:89:1322-7.

17. Arrossi S, Thouyaret L, Laudi R, Marín O, Ramírez J, Paolino M, et al. Implementation of HPV-testing for cervical cancer screening in programmatic contexts: the Jujuy demonstration project in Argentina. Int J Cancer. 2015. doi:10.1002/ijc.29530.

18. Instituto Nacional de Estadísticas y Censos (INDEC). (2012). Censo nacional de Población, Hogares y Viviendas 2010: Análisis de datos. Resultados definitivos. Cuarta publicación. Buenos Aires: INDEC. http:// www.indec.gov.ar/nivel4_default.asp?id_tema_1=2\&id_tema_2=41\&id_ tema_3=135. Accessed 06 Feb 2017.

19. WHO. Cervical cancer screening information system: Argentina. In: WHO Compendium of innovative health technologies for low-resource settings, 2011-2014. Assistive devices, eHealth solutions, Medial devices, Other technologies, Tecnologies for outbreaks. Geneve: WHO. 2015. p.84.

20. Arrossi S, Thouyaret L, Paul L. Prevención del cáncer cervicouterino. Recomendaciones para el tamizaje, seguimiento y tratamiento de mujeres en el marco de programas de tamizaje basados en el test de VPH. Actualización 2015. Buenos Aires: Instituto Nacional del Cáncer; 2015. http:// www.msal.gob.ar/images/stories/bes/graficos/0000000017cnt-manual_ recomendaciones_tamizaje_2015_baja.pdf. Accessed 08 Nov 2016.

21. Yamey $\mathrm{G}$. What are the barriers to scaling up health interventions in low and middle income countries? A qualitative study of academic leaders in implementation science. Global Health. 2012. doi:10.1186/1744-8603-8-11.

22. Alliance for Cervical Cancer Prevention (ACCP). Planning and implementing cervical cancer prevention and control programs: a manual for managers. Seattle: ACCP; 2004.

23. Gaglio B, Glasgow RE. Evaluation approaches for dissemination and implementation research. In: Brownson RC, Colditz G, Procter E, editors. Dissemination and implementation research in health. New York: Oxford University Press; 2012. p. 327-58.

24. Alvarez-Rosete A, Hawkins B, Parkhurst J. Health system stewardship and evidence informed health policy. In: Working Paper No. 1. London School of Hygiene and Tropical Medicine GRIP-Health Programme. London: GRIP-Health programme; 2013.

25. Khresheh R, Lesley B. Practice-research engagement. Jordanian experience in three Ministry of Health hospitals. Action Res. 2007. doi:10.1177/ 1476750307077313.

26. Yamey G. Scaling up global health interventions: a proposed framework for success. PLoS Med. 2011. doi:10.1371/journal.pmed.1001049.

27. Saltman RB, Ferroussier-Davis $\mathrm{O}$. The concept of stewardship in health policy. Bull World Health Organ. 2000;78:732-9.

28. Atun $\mathrm{R}$, de Jongh $\mathrm{T}$, Secci $\mathrm{F}$, Ohiri K, Adeyi O. Integration of targeted health interventions into health systems: a conceptual framework for analysis. Health Policy Plan. 2010. doi:10.1093/heapol/czp055.

29. Lehmann U, Sanders D. Community health workers: what do we know about them? The state of the evidence on programmes, activities, costs and impact on health outcomes of using community health workers. Geneva: World Health Organization; 2007

30. Strasser-Weipp K, Chavarri-Guerra Y, Virrarreal-Garza C, Bychkovsky BL, Bebiasi M, Liedke PE, et al. Progress and remaining challenges for cancer control in Latin America and the Caribbean. Lancet Oncol. 2015. doi:10. 1016/S1470-2045(15)00218-1.

31. Brownson RC, Allen P, Jacob RR, Harris JK, Duggan K, Hipp PR, et al. Understanding mis-implementation in public health practice. Am J Pre Med. 2015. doi:10.1016/j.amepre.2014.11.015.
32. Oyediran AB, Ddumba EM, Ochola SA, Lucas AO, Koporc K, Dowdle WR. A public-private partnership for malaria control: lessons from the Malarone Donation Programme. Bull World Health Organ. 2002;80:817-21.

33. IARC-WHO. IARC Handbook of Cancer Prevention. Volume 10. Cervix Cancer Screening. Lyon: WHO; 2004.

34. Arrossi S, Matos E, Zengarini N, Roth B, Sankaranayananan R, Parkin M. The socio-economic impact of cervical cancer on patients and their families in Argentina, and its influence on radiotherapy compliance. Results from a cross-sectional study. Gynecol Oncol. 2007;105:335-40.

35. Proctor $E$, Brownson R. Measurement issues in dissemination and implementation research. In: Brownson RC, Colditz G, Procter E, editors. Dissemination and implementation research in health. New York: Oxford University Press; 2012.

36. Zulu JM, Kinsman J, Michelo C, Hurtig AK. Integrating national community-based health worker programmes into health systems: a systematic review identifying lessons learned from low-and middle-income countries. BMC Public Health. 2014. doi:10.1186/1471-2458-14-987.

37. Sancho-Garnier H, Tamalet C, Halfon P, Leandri FX, Le Retraite L, Djoufelkit K, Heid P, Davies P, Piana L. HPV self-sampling or the Pap-smear: a randomized study among cervical screening nonattenders from lower socioeconomic groups in France. Int J Cancer. 2013;133(11):2681-7.

38. Arrossi S, Ramos S, Paolino M, Sankaranarayanan R. Social inequality in Pap smear coverage: identifying under-users of cervical cancer screening in Argentina. Reprod Health Matters. 2008;16(32):50-8. doi:10.1016/S09688080(08)32410-0.

39. Paolino M, Arrossi S. Analysis of the reasons for abandoning the follow-up and treatment process in women with pre-cancerous cervical lesions in the province of Jujuy: implications for health management. Salud Colect. 2012; 8(3):247-61. doi:10.1590/S1851-82652012000400003.

\section{Submit your next manuscript to BioMed Central and we will help you at every step:}

- We accept pre-submission inquiries

- Our selector tool helps you to find the most relevant journal

- We provide round the clock customer support

- Convenient online submission

- Thorough peer review

- Inclusion in PubMed and all major indexing services

- Maximum visibility for your research

Submit your manuscript at www.biomedcentral.com/submit 